\title{
Solution of the two-dimensional wave equation by using wave polynomials
}

\author{
ARTUR MACIA̧G ${ }^{*}$ AND JÖRG WAUER ${ }^{1}$ \\ Kielce University of Technology, al.1000-lecia P.P. 7, 25-314 Kielce, Poland (matam@tu.kielce.pl); ${ }^{1}$ Universität Karlsruhe, \\ Institut für Technische Mechanik, Kaiserstraße 12, D-76128 Karlsruhe, Germany (wauer@itm.uni-karlsruhe.de)
}

Received 19 November 2002; accepted in revised form 22 November 2003

\begin{abstract}
The paper demonstrates a specific power-series-expansion technique to solve approximately the two-dimensional wave equation. As solving functions (Trefftz functions) so-called wave polynomials are used. The presented method is useful for a finite body of certain shape geometry. Recurrent formulas for the wave polynomials and their derivatives are obtained in the Cartesian and polar coordinate system. The accuracy of the method is discussed and some examples are shown.
\end{abstract}

Key words: membrane vibrations, Trefftz method, wave equation, wave polynomials

\section{Introduction}

There are three groups of methods for solving partial differential equations. The first group contains the so-called analytical methods. It means that the solution exactly satisfies both the equation and all the given conditions. For example: integral-transformation method, separation-of-variables method, Green's function method and so on. Analytical solutions are not always useful for numerical calculations. For example, when the solution is given as Bessel-function series, there are often some problems with numerical convergence. Moreover, these methods are often useful only for simple body shapes. The second group contains methods such as the Finite Element Method or the Boundary Element Method. Then the solution approximately satisfies both the equation and all the given conditions and the shape does not have to be simple. In the third group the solution exactly satisfies the equation and approximately all given conditions. The Trefftz method belongs to this group. The method presented in this paper belongs to the third group. The key idea of the method is to find functions (polynomials) satisfying a given differential equation to be fitted to the governing initial and boundary conditions. In this sense it is a variant of the Trefftz method [1,2].

The method originates from [3] but only for the case of one-dimensional heat-conduction problems. Heat polynomials were applied for solving unsteady heat conduction problems [4]. Both papers describe a one-dimensional problem for the heat equation in Cartesian coordinates. The method is continued in the Cartesian coordinate system in [5, 6], describing heat polynomials for two- and threedimensional cases. Application of heat polynomials in polar and cylindrical coordinates can be found in [7-9]. A slightly different approach for one-dimensional heat polynomials is presented in [10].

In the scientific literature inverse problems are often presented. We can say that a problem is direct, when all the boundary and initial conditions known. In the inverse problems we are looking for an extrapolation of the function. The method which is described here is useful not only for direct problems but also for inverse ones. The application of this method to inverse heat-conduction problems is described in [5-9, 11, 12]. Reference [13] contains the highly interesting idea of using heat polynomials as a new type of finite-element base functions. All papers mentioned above refer to the heat-conduction 
equation. Reference [14] deals with many other cases involving other differential equations such as the Laplace, Poisson and Helmholtz equations. Also the one-dimensional wave equation is solved there. Basically, the wave equation can be solved by different methods. Some of these are better suited for infinite bodies and others for finite bodies of simple shape. The method presented here is useful for finite bodies but the shape geometry is allowed to be relatively general.

Important for the application of the method are the properties of Taylor series:

$$
\begin{aligned}
f(x+\Delta x, y+\Delta y, t+\Delta t) & =f(x, y, t) \\
& +\frac{\mathrm{d} f}{1 !}+\frac{\mathrm{d}^{2} f}{2 !}+\ldots+\frac{\mathrm{d}^{N} f}{N !}+R_{N+1}
\end{aligned}
$$

where

$$
\mathrm{d}^{n} f=\left(\frac{\partial f}{\partial x} \Delta x+\frac{\partial f}{\partial y} \Delta y+\frac{\partial f}{\partial t} \Delta t\right)^{n}
$$

and $R_{N+1}$ is the remainder term. Based on this, in Section 2 two-dimensional wave polynomials and their properties in the Cartesian coordinate system are considered. Section 3 contains two-dimensional wave polynomials in the polar coordinate system. In Section 4 the solution of the wave equation using wave polynomials is obtained. Section 5 contains some remarks on accuracy of approximation. In Section 6 some examples are discussed. Concluding remarks are given in Section 7.

\section{Wave polynomials in a Cartesian coordinate system}

There are two ways to obtain wave polynomials. The first is to use a 'generating function'. The second is to develop the wave equation in Taylor series. Both methods are equivalent and lead to the same wave polynomials.

\subsection{GENERATING FUNCTION}

Let us consider the nondimensional wave equation

$$
\frac{\partial^{2} w}{\partial t^{2}}=\frac{\partial^{2} w}{\partial x^{2}}+\frac{\partial^{2} w}{\partial y^{2}}
$$

The function

$$
g=\mathrm{e}^{\mathrm{i}(a x+b y+c t)}
$$

satisfying Equation (2) when $c^{2}=a^{2}+b^{2}$ is called a generating function for wave polynomials. The power series expansion for (3) is

$$
\mathrm{e}^{\mathrm{i}(a x+b y+c t)}=\sum_{n=0}^{\infty} \sum_{k=0}^{n} \sum_{l=0}^{n-k} S_{(n-k-l) k l}(x, y, t) a^{n-k-l} b^{k} c^{l},
$$

where $S_{(n-k-l) k l}(x, y, t)$ are polynomials of variables $x, y, t$.

Substituting $c^{2}=a^{2}+b^{2}$ in (4), we obtain

$$
\mathrm{e}^{\mathrm{i}(a x+b y+c t)}=\sum_{n=0}^{\infty} \sum_{k=0}^{n} \sum_{\substack{l=0 \\ l<2}}^{n-k} R_{(n-k-l) k l}(x, y, t) a^{n-k-l} b^{k} c^{l} .
$$


The real and imaginary parts of polynomials $R$ satisfy Equation (2) and are called wave polynomials:

$$
\begin{aligned}
& P_{(n-k-l) k l}(x, y, t)=\mathfrak{R e}\left(R_{(n-k-l) k l}(x, y, t)\right), \\
& Q_{(n-k-l) k l}(x, y, t)=\mathfrak{I m}\left(R_{(n-k-l) k l}(x, y, t)\right),
\end{aligned}
$$

e.g.

$$
\begin{aligned}
& P_{000}(x, y, t)=1, Q_{000}(x, y, t)=0, \quad P_{100}(x, y, t)=0, Q_{100}(x, y, t)=x, \\
& P_{010}(x, y, t)=0, Q_{010}(x, y, t)=y, \quad P_{001}(x, y, t)=0, Q_{001}(x, y, t)=t, \\
& P_{200}(x, y, t)=-\frac{x^{2}}{2}-\frac{t^{2}}{2}, Q_{200}(x, y, t)=0, \\
& P_{110}(x, y, t)=-x y, Q_{110}(x, y, t)=0, \quad P_{101}(x, y, t)=-x t, Q_{101}(x, y, t)=0, \\
& P_{011}(x, y, t)=-y t, Q_{011}(x, y, t)=0, \quad P_{020}(x, y, t)=-\frac{y^{2}}{2}-\frac{t^{2}}{2}, Q_{020}(x, y, t)=0, \ldots
\end{aligned}
$$

Note that there is no $R_{002}$, because $l<2$ (see formula (5)).

\subsection{PARTIAL DERIVATIVES OF WAVE POLYNOMIALS}

For numerical calculations we need the recurrent formulas of partial derivatives for wave polynomials (See section 6). To obtain these formulas we differentiate (5):

$$
\frac{\partial g}{\partial x}=\mathrm{i} a g=\sum_{n=0}^{\infty} \sum_{k=0}^{n} \sum_{\substack{l=0 \\ l<2}}^{n-k} \frac{\partial R_{(n-k-l) k l}}{\partial x} a^{n-k-l} b^{k} c^{l} .
$$

Hence

$$
\sum_{n=0}^{\infty} \sum_{k=0}^{n} \sum_{\substack{l=0 \\ l<2}}^{n-k} \mathrm{i} R_{(n-k-l) k l} a^{n-k-l+1} b^{k} c^{l}=\sum_{n=0}^{\infty} \sum_{k=0}^{n} \sum_{\substack{l=0 \\ l<2}}^{n-k} \frac{\partial R_{(n-k-l) k l}}{\partial x} a^{n-k-l} b^{k} c^{l}
$$

and

$$
\frac{\partial R_{(n-k-l) k l}}{\partial x}=\mathrm{i} R_{(n-k-l-1) k l},
$$

so that finally

$$
\frac{\partial P_{(n-k-l) k l}}{\partial x}=-Q_{(n-k-l-1) k l}, \quad \frac{\partial Q_{(n-k-l) k l}}{\partial x}=P_{(n-k-l-1) k l} .
$$

Similarly we have

$$
\frac{\partial P_{(n-k-l) k l}}{\partial y}=-Q_{(n-k-l)(k-1) l}, \quad \frac{\partial Q_{(n-k-l) k l}}{\partial y}=P_{(n-k-l)(k-1) l}
$$

and

$$
\begin{array}{ll}
\frac{\partial P_{(n-k) k 0}}{\partial t}=-Q_{(n-k-2) k 1}-Q_{(n-k)(k-2) 1}, & \frac{\partial P_{(n-k-1) k 1}}{\partial t}=-Q_{(n-k-1) k 0}, \\
\frac{\partial Q_{(n-k) k 0}}{\partial t}=P_{(n-k-2) k 1}+P_{(n-k)(k-2) 1}, & \frac{\partial Q_{(n-k-1) k 1}}{\partial t}=P_{(n-k-1) k 0} .
\end{array}
$$




\section{A. Maciag and J. Wauer}

Starting values for the derivatives (8), (9) and (10) are obtained either from (7) or directly by putting zero instead of the polynomial in question in the right-hand sides of (8), (9) and (10) if any of its subscripts takes a negative value.

\subsection{RECURRENT FORMULAS FOR WAVE POLYNOMIALS}

Recurrent formulas are useful in numerical calculations. Theorem 1 enables one to get the wave polynomials $P_{(n-k-l) k l}$ and $Q_{(n-k-l) k l}$.

THEOREM 1. Let $P_{000}=1$ and $Q_{000}=0$. Then, the polynomials

$$
\begin{aligned}
P_{(n-k) k 0} & =\frac{1}{n}\left(-x Q_{(n-k-1) k 0}-y Q_{(n-k)(k-1) 0}-t Q_{(n-k-2) k 1}-t Q_{(n-k)(k-2) 1}\right), \\
P_{(n-k-1) k 1} & =\frac{1}{n}\left(-x Q_{(n-k-2) k 1}-y Q_{(n-k-1)(k-1) 1}-t Q_{(n-k-1) k 0}\right), \\
Q_{(n-k) k 0} & =\frac{1}{n}\left(x P_{(n-k-1) k 0}+y P_{(n-k)(k-1) 0}+t P_{(n-k-2) k 1}+t P_{(n-k)(k-2) 1}\right), \\
Q_{(n-k-1) k 1} & =\frac{1}{n}\left(x P_{(n-k-2) k 1}+y P_{(n-k-1)(k-1) 1}+t P_{(n-k-1) k 0}\right),
\end{aligned}
$$

satisfy the wave Equation (2).

Proof. For relation (11) we assume that all polynomials on the right-hand side satisfy Equation (2). Substituting (11) in (2) we get

$$
\begin{aligned}
& x \underbrace{\left(\frac{\partial^{2} Q_{(n-k-1) k 0}}{\partial t^{2}}-\frac{\partial^{2} Q_{(n-k-1) k 0}}{\partial x^{2}}-\frac{\partial^{2} Q_{(n-k-1) k 0}}{\partial y^{2}}\right)}_{=0} \\
& +y(\underbrace{\frac{\partial^{2} Q_{(n-k)(k-1) 0}}{\partial t^{2}}-\frac{\partial^{2} Q_{(n-k)(k-1) 0}}{\partial x^{2}}-\frac{\partial^{2} Q_{(n-k)(k-1) 0}}{\partial y^{2}}}_{=0}) \\
& +t \underbrace{\left.\frac{\partial^{2} Q_{(n-k-2) k 1}}{\partial t^{2}}-\frac{\partial^{2} Q_{(n-k-2) k 1}}{\partial x^{2}}-\frac{\partial^{2} Q_{(n-k-2) k 1}}{\partial y^{2}}\right)}_{=0} \\
& +t \underbrace{\frac{\partial^{2} Q_{(n-k)(k-2) 1}}{\partial t^{2}}-\frac{\partial^{2} Q_{(n-k)(k-2) 1}}{\partial x^{2}}-\frac{\partial^{2} Q_{(n-k)(k-2) 1}}{\partial y^{2}}}_{=0}) \\
& +2 \frac{\partial Q_{(n-k-2) k 1}}{\partial t}+2 \frac{\partial Q_{(n-k)(k-2) 1}}{\partial t}=2 \frac{\partial Q_{(n-k-1) k 0}}{\partial x}+2 \frac{\partial Q_{(n-k)(k-1) 0}}{\partial y},
\end{aligned}
$$

whence

$$
\frac{\partial Q_{(n-k-2) k 1}}{\partial t}+\frac{\partial Q_{(n-k)(k-2) 1}}{\partial t}=\frac{\partial Q_{(n-k-1) k 0}}{\partial x}+\frac{\partial Q_{(n-k)(k-1) 0}}{\partial y}
$$

and according to (8), (9) and (10), we have

$$
P_{(n-k-2) k 0}+P_{(n-k)(k-2) 0}=P_{(n-k-2) k 0}+P_{(n-k)(k-2) 0} .
$$


This proves the theorem. The proof for (12), (13), and (14) is similar.

Similarly as before, starting values of the polynomials (11)-(14) can be obtained either from (7) or directly by putting zero instead of the polynomial in question in the right-hand side of (11)-(14) if any of its subscripts takes a negative value.

\subsection{EXPANSION OF THE WAVE EQUATION INTO TAYLOR SERIES}

Similarly as for other equations [14], the wave polynomials can be obtained using Taylor series (1) for the function $w$. Let function $w(x, y, t)$ satisfy the wave equation (2). We assume that $w \in C^{N+1}$ in the neighborhood of $\left(x_{0}, y_{0}, t_{0}\right)$. Let $\hat{x}=x-x_{0}, \hat{y}=y-y_{0}, \hat{t}=t-t_{0}$. Then, the Taylor series for function $w$ and for $\mathrm{N}=2$ is

$$
\begin{aligned}
w(x, y, t)= & w\left(x_{0}, y_{0}, t_{0}\right)+\frac{\partial w}{\partial x} \hat{x}+\frac{\partial w}{\partial y} \hat{y}+\frac{\partial w}{\partial t} \hat{t}+\frac{\partial^{2} w}{\partial x^{2}} \frac{\hat{x}^{2}}{2}+\frac{\partial^{2} w}{\partial y^{2}} \frac{\hat{y}^{2}}{2}+\frac{\partial^{2} w}{\partial t^{2}} \frac{\hat{t}^{2}}{2} \\
& +\frac{\partial^{2} w}{\partial x \partial y} \hat{x} \hat{y}+\frac{\partial^{2} w}{\partial x \partial t} \hat{x} \hat{t}+\frac{\partial^{2} w}{\partial y \partial t} \hat{y} \hat{t}+R_{3} .
\end{aligned}
$$

Eliminating the derivative $\partial^{2} w / \partial t^{2}$ by Equation (2) yields

$$
\begin{aligned}
w(x, y, t)= & w\left(x_{0}, y_{0}, t_{0}\right)+\frac{\partial w}{\partial x} \hat{x}+\frac{\partial w}{\partial y} \hat{y}+\frac{\partial w}{\partial t} \hat{t}+\frac{\partial^{2} w}{\partial x^{2}}\left(\frac{\hat{x}^{2}}{2}+\frac{\hat{t}^{2}}{2}\right) \\
& +\frac{\partial^{2} w}{\partial y^{2}}\left(\frac{\hat{y}^{2}}{2}+\frac{\hat{t}^{2}}{2}\right)+\frac{\partial^{2} w}{\partial x \partial y} \hat{x} \hat{y}+\frac{\partial^{2} w}{\partial x \partial t} \hat{x} \hat{t}+\frac{\partial^{2} w}{\partial y \partial t} \hat{y} \hat{t}+R_{3} .
\end{aligned}
$$

The coefficients succeeding the derivation terms on the right-hand side represent the non-zero wave polynomials (7). Similarly, we get polynomials for $N=3,4, \ldots$.

\section{Wave polynomials in polar coordinates}

To obtain wave polynomials in polar coordinates,

$$
x=r \cos \phi, y=r \sin \phi,
$$

we substitute (17) in (2) to get

$$
\frac{\partial^{2} w}{\partial t^{2}}=\frac{\partial^{2} w}{\partial r^{2}}+\frac{1}{r} \frac{\partial w}{\partial r}+\frac{1}{r^{2}} \frac{\partial^{2} w}{\partial \phi^{2}} .
$$

Then, to find the wave polynomials in polar coordinates, it is sufficient to substitute (17) in polynomials expressed in the Cartesian coordinate system. The polynomials obtained in that way satisfy Equation (18), e.g., for the non-zero polynomials (7)

$$
\begin{aligned}
P_{000}(r, \phi, t) & =1, \quad Q_{100}(r, \phi, t)=r \cos \phi, \\
Q_{010}(r, \phi, t) & =r \sin \phi, \quad Q_{001}(r, \phi, t)=t, \\
P_{200}(r, \phi, t) & =-\frac{r^{2} \cos ^{2} \phi}{2}-\frac{t^{2}}{2}, P_{110}(r, \phi, t)=-r^{2} \cos \phi \sin \phi, \ldots
\end{aligned}
$$

We can use the recurrent formulas (8)-(10) and (11)-(14) also in polar coordinates, keeping in mind that $x=r \cos \phi$ and $y=r \sin \phi$. 


\section{Wave-polynomial method}

The wave-polynomial method discussed below belongs to the class of Trefftz methods. As a solution of wave Equation (2) we take a linear combination of wave polynomials which also satisfies Equation (2).

Let us denote the non-zero polynomials as

$$
\begin{gathered}
V_{1}=1, V_{2}=x, V_{3}=y, V_{4}=t, V_{5}=-\frac{x^{2}}{2}-\frac{t^{2}}{2}, V_{6}=-x y, \\
V_{7}=-x t, V_{8}=-y t, V_{9}=-\frac{y^{2}}{2}-\frac{t^{2}}{2}, \ldots
\end{gathered}
$$

Obviously, we have one polynomial of order zero, three polynomials of order one, five polynomials of order two and so on.

An approximation for the solution of Equation (2) is

$$
w \approx u=\sum_{n=1}^{N} c_{n} V_{n} .
$$

Because all polynomials $V_{n}$ satisfy Equation (2), also the linear combination $u$ satisfies Equation (2). The coefficients $c_{n}$ in (21) are chosen such that the error for fulfilling given boundary and initial conditions corresponding to Equation (2) is minimized (see examples).

\section{Accuracy of approximation}

The wave-polynomial method is an approximation method. It is very important to know how big is the error of approximation. Moreover, this method should be convergent. It is easy to specify the error when in formula (21) all polynomials from order zero to $K$ are taken, e.g., for $K=0, N=1$, for $K=1, N=1+3=4$, for $K=2, N=1+3+5=9$ and so on. Then the error of approximation is equal to the remainder term in the Taylor series for function $w$ (see relations (15) and (16)). This means that the wave-polynomial method is convergent if

$$
\lim _{N \rightarrow \infty} R_{N}=0
$$

in the Taylor series of function $w$.

\section{Examples}

We will show how the wave-polynomial method works by discussing two easy examples. The first concerns the vibrations of a square membrane. The second discusses the vibrations of a circular membrane.

\subsection{VibRations of A SQUARE MEMBrane}

Let us consider the free vibrations of square membranes described by the following mathematical model:

- equation of motion:

$$
\frac{\partial^{2} w}{\partial t^{2}}=\frac{\partial^{2} w}{\partial x^{2}}+\frac{\partial^{2} w}{\partial y^{2}} \quad(x, y) \in(0,1) \times(0,1), t>0,
$$


- initial conditions:

$$
w(x, y, 0)=w_{0}(x, y)=x(x-1) y(y-1), \quad \frac{\partial w(x, y, 0)}{\partial t}=0,
$$

- boundary conditions:

$w(0, y, t)=w(1, y, t)=w(x, 0, t)=w(x, 1, t)=0$.

The exact solution of this problem is; (see [15, pp. 293-296])

$$
w(x, y, t)=\frac{16}{\pi^{2}} \sum_{k, l=1}^{\infty} \frac{\left[(-1)^{k}-1\right]\left[(-1)^{l}-1\right] \sin (k \pi x) \sin (l \pi y) \cos \left(\sqrt{k^{2}+l^{2}} \pi t\right)}{k^{3} l^{3}} .
$$

All results below are obtained for $k, l=1, \ldots, M$ where $M=40$. The difference between the solutions for $M=41$ and $M=40$ is less than $5 \times 10^{-6}$ in terms of the maximum norm.

The solution $w(x, y, t)$ is approximated according to Equation (21):

$$
w \approx u=\sum_{n=1}^{N} c_{n} V_{n}
$$

Because the function $u$ satisfy the wave equation (linear combination of wave polynomials), we minimize $\|w-u\|$ for the boundary and initial conditions. We look for an approximate solution $u$ in the time interval $(0, \Delta t)$. Therefore, the coefficients $c_{n}$ have to be chosen appropriately to minimize the functional

$$
\begin{aligned}
I & =\underbrace{\int_{0}^{1} \int_{0}^{1}\left[u(x, y, 0)-w_{0}(x, y)\right]^{2} \mathrm{~d} y \mathrm{~d} x}_{\text {cond. (23) }}+\underbrace{\int_{0}^{1} \int_{0}^{1}\left[\frac{\partial u(x, y, 0)}{\partial t}-\frac{\partial w(x, y, 0)}{\partial t}\right]^{2} \mathrm{~d} y \mathrm{~d} x}_{\text {cond. }(23)} \\
& +\underbrace{\int_{0}^{\Delta t} \int_{0}^{1}[u(0, y, t)-w(0, y, t)]^{2} \mathrm{~d} y \mathrm{~d} t}_{\text {cond. }}+\underbrace{\int_{0}^{\Delta t} \int_{0}^{1}[u(1, y, t)-w(1, y, t)]^{2} \mathrm{~d} y \mathrm{~d} t}_{\text {cond. }} \\
& +\underbrace{\int_{0}^{\Delta t} \int_{0}^{1}[u(x, 0, t)-w(x, 0, t)]^{2} \mathrm{~d} x \mathrm{~d} t}_{\text {cond }}+\underbrace{\int_{0}^{\Delta t} \int_{0}^{1}[u(x, 1, t)-w(x, 1, t)]^{2} \mathrm{~d} x \mathrm{~d} t} .
\end{aligned}
$$

A necessary condition to minimize the functional $I$ is

$$
\frac{\partial I}{\partial c_{1}}=\ldots=\frac{\partial I}{\partial c_{N}}=0 .
$$

The linear system of Equations (27) can be written as

$$
A C=B
$$

where $C=\left[c_{1}, \ldots, c_{N}\right]^{T}$ and the elements of matrices $A$ and $B$ are

$$
\begin{aligned}
a_{i j}= & \int_{0}^{1} \int_{0}^{1} V_{i}(x, y, 0) V_{j}(x, y, 0) \mathrm{d} y \mathrm{~d} x+\int_{0}^{1} \int_{0}^{1} \frac{\partial V_{i}(x, y, 0)}{\partial t} \frac{\partial V_{j}(x, y, 0)}{\partial t} \mathrm{~d} y \mathrm{~d} x \\
& +\int_{0}^{\Delta t} \int_{0}^{1} V_{i}(0, y, t) V_{j}(0, y, t) \mathrm{d} y \mathrm{~d} t+\int_{0}^{\Delta t} \int_{0}^{1} V_{i}(1, y, t) V_{j}(1, y, t) \mathrm{d} y \mathrm{~d} t
\end{aligned}
$$



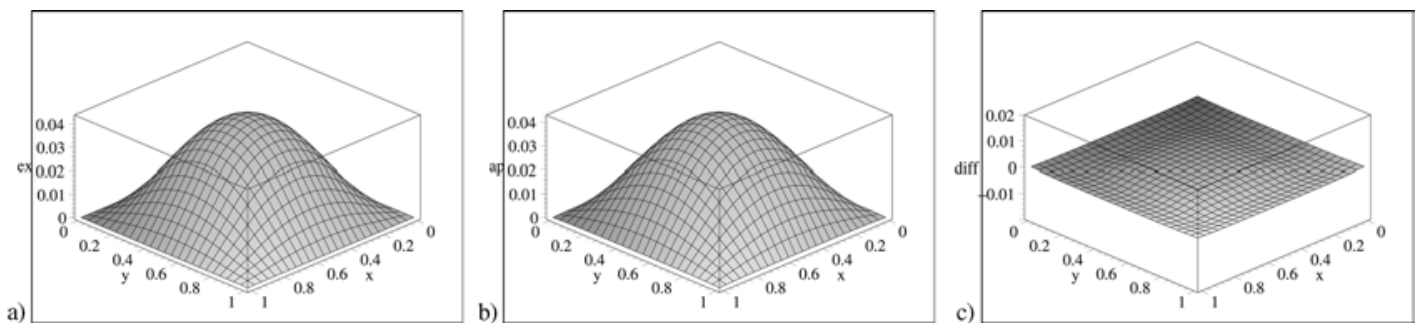

Figure 1. Solution for time $t=0 \cdot 2$ : a) exact, b) approximation, c) difference.
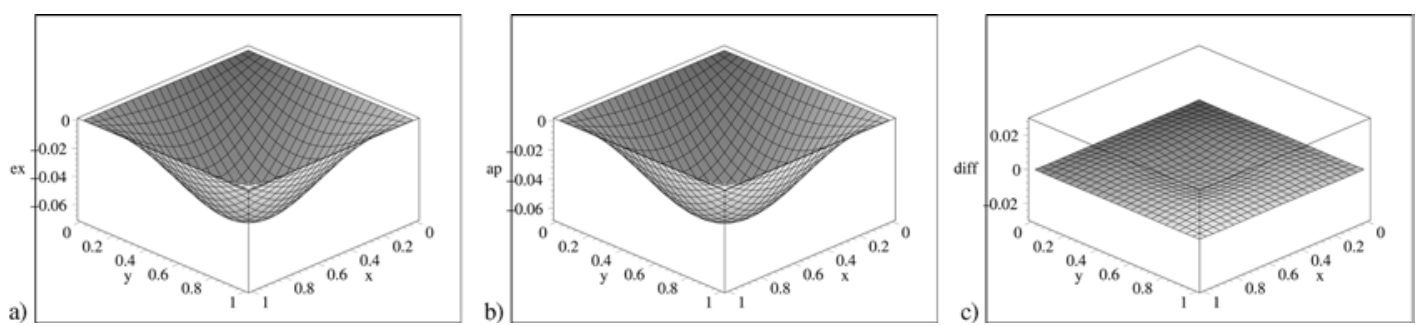

Figure 2. Solution for time $t=0 \cdot 7$ : a) exact, b) approximation, c) difference.

$$
\begin{aligned}
& +\int_{0}^{\Delta t} \int_{0}^{1} V_{i}(x, 0, t) V_{j}(x, 0, t) \mathrm{d} x \mathrm{~d} t+\int_{0}^{\Delta t} \int_{0}^{1} V_{i}(x, 1, t) V_{j}(x, 1, t) \mathrm{d} x \mathrm{~d} t, \\
b_{i}= & \int_{0}^{1} \int_{0}^{1} V_{i}(x, y, 0) w_{0}(x, y) \mathrm{d} y \mathrm{~d} x .
\end{aligned}
$$

Note that $a_{i j}=a_{j i}$ (matrix $A$ is symmetric). This fact facilitates the calculations. From Equation (28) we obtain the coefficients $c_{n}$ :

$$
C=A^{-1} B \text {. }
$$

In the time intervals $(\Delta t, 2 \Delta t),(2 \Delta t, 3 \Delta t), \ldots$, we proceed analogously. Here, the initial condition for time interval $((m-1) \Delta t, m \Delta t)$ is the value of function $u$ at the end of interval $((m-2) \Delta t,(m-1) \Delta t)$. All results below have been obtained for $\Delta t=1$.

We obtain a good approximation in whole time interval $(0, \Delta t)$. For example, Figure 1 shows for time $t=0 \cdot 2$ a) the exact solution, b) an approximation by polynomials from order 0 to 9 , c) the difference between a) and $b$ ).

Figure 2 shows for time $t=0 \cdot 7$ : a) the exact solution, b) an approximation by polynomials from order 0 to 9 , c) the difference between a) and b). It is obvious that the approximation presented in the Figures 1 and 2 is very accurate. Figure 3 shows the exact result for the vibration as a function of time for the location $x=0.5, y=0.5$ and the approximation by polynomials from order 0 to a) $4, \mathrm{~b}$ ) $6, \mathrm{c}$ ) 9.

In this case we can calculate the error of approximation in the norm $H^{1}(\Omega)$, where $\Omega=(0, \Delta t)$ for point $x=y=0.5$. The error is defined as:

$$
\text { Error }=\sqrt{\frac{\int_{0}^{\Delta t}[w(0 \cdot 5 ; 0.5 ; t)-u(0.5 ; 0 \cdot 5 ; t)]^{2} \mathrm{~d} t}{\int_{0}^{\Delta t}[w(0 \cdot 5 ; 0.5 ; t)]^{2} \mathrm{~d} t}} .
$$

In approximation $u$ we take all wave polynomials from order 0 to $K$. Table 1 shows the error which depends on the order $K$. 

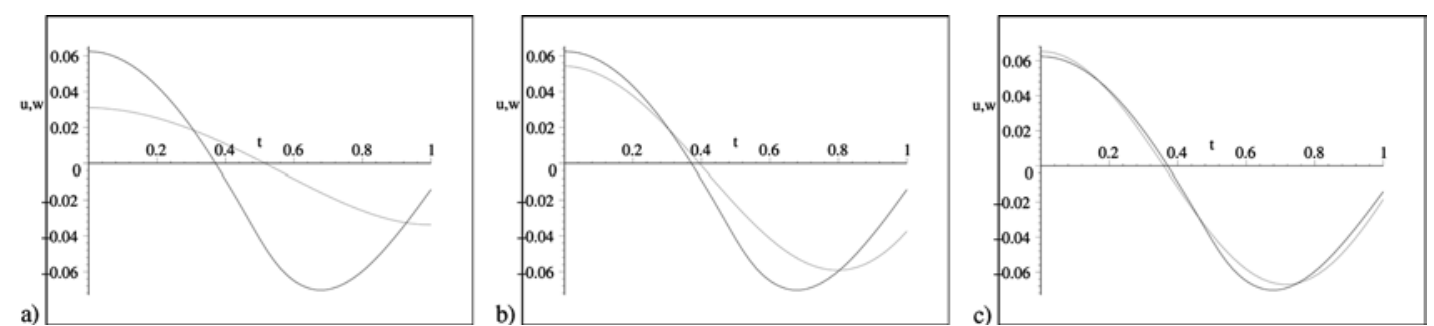

Figure 3. Exact solution versus time for point $x=0.5, y=0.5$ and approximation by polynomials from order 0 to a) 4 , b) 6 , c) 9.

Table 1. Error dependence of the polynomial order.

\begin{tabular}{ll}
\hline Order $K$ & Error \\
\hline 4 & 0.6836 \\
6 & 0.2845 \\
9 & 0.0772 \\
\hline
\end{tabular}

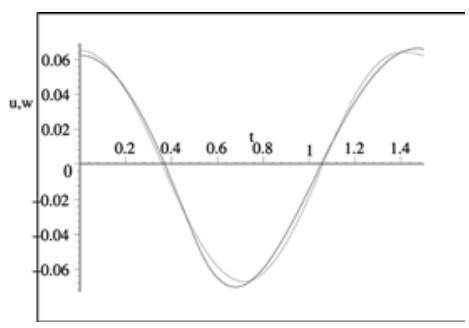

Figure 4. Extrapolation in time.

The error decreases when the number of the polynomials in approximation $u$ increases. This means that the wave-polynomials method is convergent.

The wave-polynomials method is also useful for inverse problems. This means that we need an extrapolation of approximation $u$ either in time or in space. The results are obtained for $\Delta t=1$. Figure 4 shows the extrapolation in time for the vibration of point $x=0.5, y=0.5$ by polynomials up to order 9. Again it is obvious that the approximation and the extrapolation in time coincide very well.

\subsection{VIBRATION OF A CIRCULAR MEMBRANE}

Let us consider free vibrations of a circular membrane described (in Cartesian coordinates) by the following mathematical model:

- equation of motion:

$$
\frac{\partial^{2} w}{\partial t^{2}}=\frac{\partial^{2} w}{\partial x^{2}}+\frac{\partial^{2} w}{\partial y^{2}}, \quad x^{2}+y^{2}<1, \quad t>0,
$$

- initial conditions:

- a boundary condition:

$$
w(x, y, 0)=w_{0}(x, y)=\frac{1}{20}\left(1-x^{2}-y^{2}\right), \quad \frac{\partial w(x, y, 0)}{\partial t}=0,
$$

$$
w(x, y, t)=0, \quad x^{2}+y^{2}=1 .
$$

Transforming the problem into polar coordinates, we get

- equation of motion:

$$
\frac{\partial^{2} w}{\partial t^{2}}=\frac{\partial^{2} w}{\partial r^{2}}+\frac{1}{r} \frac{\partial w}{\partial r}+\frac{1}{r^{2}} \frac{\partial^{2} w}{\partial \phi^{2}}, \quad(r, \phi) \in(0,1) \times(0,2 \pi), \quad t>0
$$


- initial conditions:

$$
w(r, \phi, 0)=w_{0}(r, \phi)=\frac{1-r^{2}}{20}, \quad \frac{\partial w(r, \phi, 0)}{\partial t}=0,
$$

- a boundary condition:

$$
w(1, \phi, t)=0,
$$

The exact solution for this problem, using Bessel functions $J_{0}$ and $J_{1}$, is; (see [15, pp. 296-298])

$$
w(r, t)=\sum_{n=1}^{\infty} \frac{J_{0}\left(k_{n} r\right) \cos \left(k_{n} t\right)}{10 J_{1}^{2}\left(k_{n}\right)} \int_{0}^{1} r\left(1-r^{2}\right) J_{0}\left(k_{n} r\right) \mathrm{d} r,
$$

where $k_{n}$ are positive roots of the equation $J_{0}(k)=0$. Unfortunately, for a large number of these functions in the polar coordinate system there are some problems with convergence.

Let us use the solution (21):

$$
w \approx u=\sum_{n=1}^{N} c_{n} V_{n},
$$

where $V_{n}$ are the wave polynomials in polar coordinates. We look for an approximate solution in the time interval $(0, \Delta t)$. The coefficients $c_{n}$ have to be computed such that the functional

$$
\begin{aligned}
I & =\underbrace{\int_{0}^{1} \int_{0}^{2 \pi}\left[u(r, \phi, 0)-w_{0}(r, \phi)\right]^{2} r \mathrm{~d} \phi \mathrm{d} r}_{\text {cond.(33) }}+\underbrace{\int_{0}^{1} \int_{0}^{2 \pi}\left[\frac{\partial u(r, \phi, 0)}{\partial t}-\frac{\partial w(r, \phi, 0)}{\partial t}\right]^{2} r \mathrm{~d} \phi \mathrm{d} r}_{\text {cond.(33) }} \\
& +\underbrace{\int_{0}^{\Delta t} \int_{0}^{2 \pi}[u(1, \phi, t)-w(1, \phi, t)]^{2} r \mathrm{~d} \phi \mathrm{d} t}_{\text {cond.(34) }}
\end{aligned}
$$

is minimized. To obtain the coefficients $c_{n}$, the procedure is the same as in Section 6.1. Here the elements of the matrices $A$ and $B$ are:

$$
\begin{aligned}
a_{i j} & =\int_{0}^{1} \int_{0}^{2 \pi} V_{i}(r, \phi, 0) V_{j}(r, \phi, 0) r \mathrm{~d} \phi \mathrm{d} r+\int_{0}^{1} \int_{0}^{2 \pi} \frac{\partial V_{i}(r, \phi, 0)}{\partial t} \frac{\partial V_{j}(r, \phi, 0)}{\partial t} r \mathrm{~d} \phi \mathrm{d} r \\
& +\int_{0}^{\Delta t} \int_{0}^{2 \pi} V_{i}(1, \phi, t) V_{j}(1, \phi, t) r \mathrm{~d} \phi \mathrm{d} t \\
b_{i} & =\int_{0}^{1} \int_{0}^{2 \pi} V_{i}(r, \phi, 0) w_{0}(r, \phi) r \mathrm{~d} \phi \mathrm{d} r .
\end{aligned}
$$

Similarly as in Section 6.1 matrix $A$ is symmetric.

All results below are obtained for $\Delta t=1$. In this case the solution is independent of the angle $\phi$. Figure 5 shows the vibrations as dependent on radius and time: a) the exact solution, b) an approximation by polynomials from order 0 to 9 , c) the difference between a) and b). It is obvious that the approximation result is very good.

Figure 6 shows the exact solution of the vibration versus time for the midpoint $r=0$ and an approximation by polynomials from order 0 to a) 3, b) 5 , c) 9 . We can calculate the error of approximation in the norm $H^{1}(\Omega)$, where $\Omega=(0, \Delta t)$ for point $r=0$. The error is defined as: 

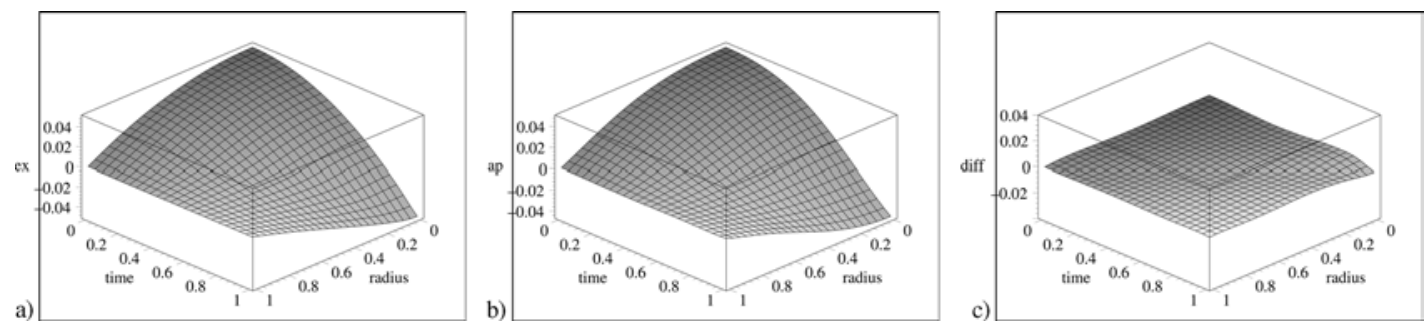

Figure 5. Solution versus time $t \in[0,1]$ : a) exact, b) approximation, c) difference.
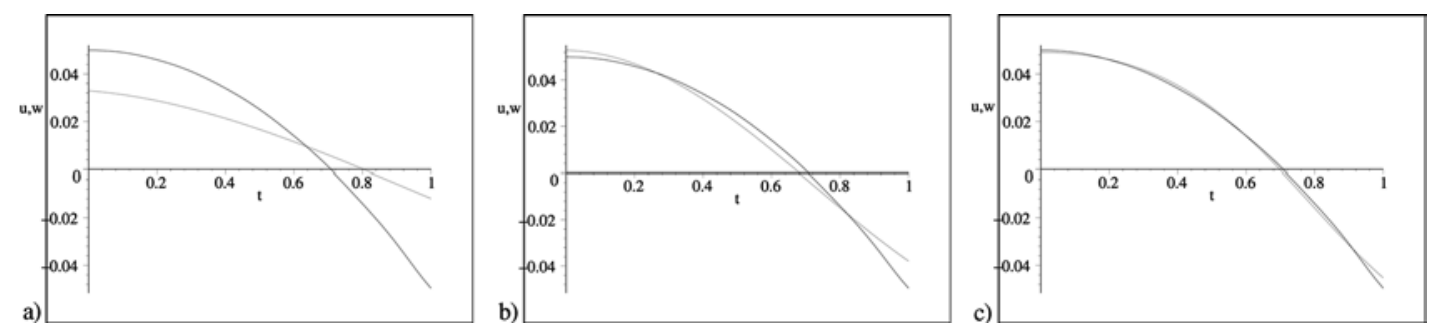

Figure 6. Vibration versus time for point $r=0$ by polynomials from order 0 to: a) 3 , b) 5 , c) 9 .

Table 2. Error dependence of the polynomial order.

\begin{tabular}{ll}
\hline Order $K$ & Error \\
\hline 4 & 0.4848 \\
6 & 0.1059 \\
9 & 0.0361 \\
\hline
\end{tabular}

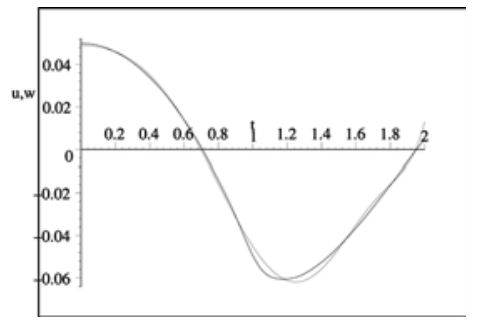

Figure 7. Extrapolation in time.

$$
\text { Error }=\sqrt{\frac{\int_{0}^{\Delta t}[w(r, \phi, t)-u(r, \phi, t)]^{2} \mathrm{~d} t}{\int_{0}^{\Delta t}[w(r, \phi, t)]^{2} \mathrm{~d} t} .}
$$

Here the angle $\phi$ is arbitrary $(e . g \phi=0)$, because the solution is independent of the angle. In the approximation $u$ we take all wave polynomials from order 0 to $K$. Table 1 shows the error dependence of order $K$. Obviously, the error decreases when the number of the polynomials in approximation $u$ increases. It means that the wave-polynomials method is convergent in polar coordinate system.

The extrapolation of approximation $u$ can be used also in polar coordinates. Figure 7 shows the extrapolation in time for the vibration of point $r=0$ by polynomials up to order 9 (the results are obtained for $\Delta t=1)$.

Again, it is obvious that approximation and extrapolation in time coincide very well.

\section{Concluding remarks}

A new technique for solving the two-dimensional wave equation has been developed. Our main result is the derivation of the formulas for the wave polynomials (satisfying a wave equation) and their derivatives. The wave-polynomial method presented in this paper is a straightforward method for solving wave equations in finite bodies. This method is also useful when the shape of the body is complicated. 
The only condition is that the shape geometry is given or can be approximated in polynomial form. Then, the evaluation basically remains unchanged and the only difficulty for such a shape may be the calculation of the integrals determining the coefficients $c_{n}$ - for most shapes this does not create any problems. The method is convergent and the error is equal to the remainder term in the Taylor series. The simple examples presented show that the obtained approximations of the exact solutions are very good, both in Cartesian and polar coordinates. Especially, in the polar coordinate system this method avoids ill-convergent Bessel functions. The solution, being a linear combination of wave polynomials, exactly satisfies the wave equation and approximately initial and boundary conditions. It is important, that this method can be used for extrapolation. Therefore, the wave-polynomial method can also be applied to inverse problems. Wave polynomials can be used as finite-element base functions. This will be the subject of another paper.

\section{Acknowledgements}

A. Maciagg is an award holder of the NATO Science Fellowships Programme. He wishes to thank also the University of Karlsruhe where the paper was written.

\section{References}

1. E. Trefftz, Ein Gegenstük zum Ritzschen Verfahren. In: Proceedings 2nd International Congres of Applied Mechanics. Zurich (1926) pp. 131-137.

2. A.P. Zieliński and I. Herrera, Trefftz method: fitting boundary conditions. Int. J. Num. Meth. Engng. 24 (1987) 871-891.

3. P.C. Rosenbloom and D.V. Widder, Expansion in terms of heat polynomials and associated functions. Trans. Am. Math. Soc. 92 (1956) 220-266.

4. H. Yano, S. Fukutani and A. Kieda, A boundary residual method with heat polynomials for solving unsteady heat conduction problems. J. Franklin Inst. 316 (1983) 291-298.

5. S. Futakiewicz and L. Hożejowski, Heat polynomials method in the n-dimensional direct and inverse heat conduction problems. In: A.J.Nowak, C.A.Brebbia, R.Bielecki and M.Zerroukat (eds.), Advanced Computational Method in Heat Transfer V. Southampton, UK and Boston, USA: Computational Mechanics Publications (1998) pp. 103-112.

6. L. Hożejowski, Heat Polynomials and their Application for Solving Direkt and Inverse Heat Condutions Problems (PhDThesis). Kielce: University of Technology (1999) 115 pp. (in Polish)

7. S. Futakiewicz and L. Hożejowski, Heat polynomials in solving the direct and inverse heat conduction problems in a cylindrical system of coordinates. In: A.J.Nowak, C.A.Brebbia, R.Bielecki and M.Zerroukat (eds.), Advanced Computational Method in Heat Transfer V. Southampton UK and Boston USA: Computational Mechanics Publications (1998) pp. 71-80.

8. S. Futakiewicz, K. Grysa and L. Hożejowski, On a problem of boundary temperature identification in a cylindrical layer. In: B.T.Maruszewski, W.Muschik and A.Radowicz (eds.), Proceedings of the International Symposium on Trends in Continuum Physics. Singapore, New Jersey, London, Hong Kong: World Scientific Publishing (1999) pp. 119-125.

9. S. Futakiewicz, Heat Functions Method for Solving Direkt and Inverse Heat Condutions Problems (PhD-Thesis). Poznań: University of Technology (1999) 120 pp. (in Polish)

10. P. Johansen, M. Nielsen and O.F. Olsen, Branch points in one-dimensional Gaussian scale space. Math. Imag. Vision 13 (2000) 193-203.

11. M.J. Ciałkowski, S. Futakiewicz and L. Hożejowski, Method of heat polynomials in solving the inverse heat conduction problems. ZAMM 79 (1999) 709-710.

12. M.J. Ciałkowski, S. Futakiewicz and L. Hożejowski, Heat polynomials applied to direct and inverse heat conduction problems. In: B.T.Maruszewski, W.Muschik and A.Radowicz (eds.), Proceedings of the International Symposium on Trends in Continuum Physics. Singapore, New Jersey, London, Hong Kong: World Scientific Publishing (1999) pp. 79-88.

13. M.J. Ciałkowski, Solution of inverse heat conduction problem with use new type of finite element base functions. In: B.T. Maruszewski, W. Muschik and A. Radowicz (eds.), Proceedings of the International Symposium on Trends in Continuum Physics. Singapore, New Jersey, London, Hong Kong: World Scientific Publishing (1999) pp. 64-78.

14. M.J. Ciałkowski and A. Frąckowiak, Heat Functions and Their Application for Solving Heat Transfer and Mechanical Problems. Poznań: University of Technology Publishers (2000) 360 pp. (in Polish)

15. I.N. Sneddon, Elements of Partial Differential Equations. Warsaw: PWN (1962) 423 pp. 